\title{
A day-to-day experiment on an urban road network in ordinary and emergency scenarios
}

\author{
M. L. De Maio, G. Musolino \& A. Vitetta \\ DIIES - Dipartimento di ingegneria dell'Informazione, \\ delle Infrastrutture e dell'Energia Sostenibile, \\ Università degli Studi Mediterranea di Reggio Calabria, Italy
}

\begin{abstract}
Users' perception of the network plays a key role in the way users behave. In this paper the attention is focused on choice set formation and route choices, trying to represent their day-to-day evolution. Both experience of the network and information acquired influence users' perception. In order to investigate this issue, a day-to-day experiment is carried out on a real road network. Results have been elaborated and some descriptive analyses are presented. The results have a particular significance in the case of evacuation where the information, in smart cities, is defined and provided according to forecasted users' behaviour.

Keywords: route choice, switching behaviour, day-to-day models, information, evacuation, smart city.
\end{abstract}

\section{Introduction}

In a road transportation system, if users are made aware of the real situation currently occurring on the network by means of an information system in smart cities, they can use the information acquired in the choice process and eventually change their mind, modifying habitual choices and also the perceived choice set. For this reason, the main purpose of this paper consists of analysing the role of information diffused and the way users' behaviour is influenced and eventually changed, with a real experimentation.

Some simulation models, consolidated in literature, assumed that users are perfectly and immediately aware of changes on the network and that users perceive the entire set of path alternatives available. Recently, some researchers 
underlined that this assumption is too restrictive and they showed that users perceive a little number of alternatives [1]. Usually users have not a complete knowledge of current network conditions and users' choices are based on their network perception, which may or not reflect the real situation. Information gives to users a more correct perception of current network conditions and makes them aware of changes on the network. Users' previous knowledge of the network, the type of information received and information reliability influences their compliance to information received.

User's perception of travel time is likely influenced by travel times experienced on the network in the past. Therefore, choices of the current day depend on choices made in the past. Day-to-day models have been used in literature in order to capture choices evolution [2]. The introduction of ATIS increased information availability and modified the way users make their choices [3]. The issues of convergence towards a certain attractor and stability of equilibrium are addressed in several works $[4,5]$. Calibration of a day-to-day model requires the use of panel data which may be difficult to be collected and correctly analysed.

If compared to ordinary conditions, providing information to users in emergency conditions becomes a hard task. This because some ATIS (Advanced Traveller Information Systems) used in ordinary conditions, may not work in emergency conditions [6]. The recent widespread of new technologies changed the way information are conveyed. In addition to this, it was showed that both the type of informer [7], who provides information, and the previous training [8] influence users' reactions. Many models and methods for ordinary conditions lose their applicability in emergency conditions. For this reason researchers dealt with emergency conditions reconsidering every component of the transportation system: travel demand in evacuation conditions was treated in [9], whereas transportation supply and traffic assignment were dealt in [10] and [11]. Moreover, route choice models in evacuation conditions were classified in [12] and the issue of dynamic choices in emergency conditions was treated in $[13-15]$.

As a consequence of change in users' perception, both alternatives perceived (belonging to the choice set) and alternative chosen may change. Many works available in literature assumed that users' choice set is fixed, as it is usually assumed in the static context. On the contrary, in this work, choice set evolution is allowed and the way it occurs day-to-day is investigated.

In order to collect data, laboratory experiments are frequently performed. A laboratory-like experiment was carried out in [16]: the interviewed users were asked to choose between two alternative routes for twenty days. Different information scenarios were proposed. No specific trends to an equilibrium state were shown during the experiment. Both experience and information were involved in the simulation of a learning process in [17]. Perception of travel times and reliability of information are explicitly modelled by means of a day-today dynamic framework. In Mahmassani and Liu [18], the influence of ATIS and experience on both route choice and departure time was simulated. The experimentation is developed in laboratory over five days and carried out using a 
travel simulator: users, provided with real time information, make their choices simultaneously on a simulated traffic corridor. Recently, Meneguzzer and Olivieri [19] realized a laboratory experiment over a long period (50 days). Thirty interviewed users were provided only with feedback information on the route chosen the day before. The results showed that user equilibrium does not persist.

In this paper a day-to-day experiment, carried out on a real network, is presented and some descriptive statistics are reported. This kind of experiment may be successfully conducted in a smart city (defined in the report "Smart cities - Ranking of European medium-sized cities" [20] because of the ICTinfrastructure implemented. The experiment is designed in order to capture both choice set evolution and route choice evolution, taking into account the influence of information provided to users. Several information scenarios are proposed in order to analyse different users' reactions. The experiment gives us insights about the real process of perception evolution, which is the first step necessary to specify a mathematical model of perception.

The following part of the paper is composed of four sections. The experiment is presented in section 2 and the main results obtained are commented by means of the descriptive statistics in section 3. Conclusions and further developments are summarized in section 4 .

\section{The experiment}

The main goals of the day-to-day route switching experiment are the analysis of: (i) choice set formation and evolution; (ii) influence of information on alternatives perception and route choice evolution.

\section{Choice set formation and evolution}

The experiment is conceived as a day-to-day experiment in order to capture the day-to-day evolution of choice set and route choice. Considering that choices are individual and that reaction to information received may be different amongst users, the experiment is disaggregated. In other words each user is interviewed separately and is characterized by a series of choices and a perceived choice set.

The experiment was carried out in the city of Reggio Calabria during the period of May 2013. The sample consists of fifteen people and was developed in six days.

In order to have a more complete knowledge of the users composing the sample, three questions were asked during the experiment. The first question was about users' knowledge of the network. A five values scale was used to measure it: an excellent knowledge of the network corresponds to a value equal to five, whereas a low knowledge of the network corresponds to a value equal to one. The second question was proposed to get more insights on the effective use of the road network: users were asked if they had a private mean of transport available. The third question gave us information about users' age.

On each day of the experiment period, users of the sample were interviewed about the route chosen. The area of the experiment is a well-known central part of the city. The origin and the destination of the trip were fixed. 
The origin was located close to the Museum of Magna Graecia of Reggio Calabria (localized in the CBD) and the destination was at the Engineering Faculty of University Mediterranea of Reggio Calabria (localized in the suburb and distant about one chilometre from the origin. For the origin and the destination it was indicated an exact point and not a zone (as commonly used in other choice dimensions). The indication of an exact point derived from the consideration that the position influences route choice and perceived alternatives.

In order to analyse choice set formation, in the first day, users were asked to identify their preferred route between the proposed origin-destination pair. In addition, they were asked to think if there was/were another/others route/s they were considering in their choice. After a few days of interviews, users were asked again if they were considering also some other routes. It was found that, after a few days of experiment, users perceived one or two additional routes than the chosen/perceived alternatives up to that day. This may be explained considering two main elements:

- users, being asked to choose every day a route on the given origin-destination pair, consider past experience and a map;

- users exchanged opinions with each other: indeed some users, during the experiment, choose or perceive also some paths that were not experienced, perceived or indicated before.

It is worth underlying that in some other experiments cooperation is considered absent. The results obtained in this experiment show that this assumption is too restrictive.

\section{Route choice influenced by information and route switching}

The experiment was designed in order to create three different scenarios related to information supplied to users. For this aim, the sample was subdivided into three groups: all levels of network knowledge were represented in each group. The joint group is also analysed. Users do not know that they belonged to a specific group of experiment. The main differences between the groups are explained:

- Group 1 "no information": users receive information about the travel time experienced on the route chosen the day before;

- Group 2 "right information": users receive information about the travel time on the chosen route and they are provided with additional right information on an alternative route;

- Group 3 "wrong information": users receive information about the travel time on the chosen route and they are provided with additional wrong information on an alternative route.

- Joint Group: obtained considering the entire sample.

All users were provided with information about the travel time experienced on the route chosen the day before. Users of the second and third group were provided with additional information on an alternative route. In particular, users of the second group received correct information about the current travel time on the alternative route; whereas users of the third group received wrong information about the current travel time on the alternative route. In this way, we 
could analyse both the influence of different kinds of information on users and the role played by information reliability.

Travel times on routes are calculated by means of a simulation tool able to reproduce flows and congested travel times on the road network of the town of Reggio Calabria. Moreover, it is assumed that, when the user receives information on an alternative route (also relative to the quality of the road [21], the alternative route will enter in his choice set from then on. This does not mean that the user will choose the new alternative.

If the user changes his mind and he decides not to choose the same route chosen the day before, there is a switching. The frequency of the switching behaviour may be influenced by user's knowledge of the network, information obtained day-to-day and information reliability.

\section{Results analysis}

In this section some descriptive statistics concerning the survey are reported. The additional questions on users' characteristics could help to explain the results. Section three is composed of three subsections: the first reports comments of the results obtained in relation to the choice set formation and evolution; the second reports choice evolution; the third is devoted to switching behaviour.

\subsection{Choice set formation and evolution}

Users of the sample identified five routes on the road network during the experiment. Therefore, the joint choice set in this experiment is composed of five routes. These routes are called with the labels: $\mathrm{k} 1 ; \mathrm{k} 2 ; \mathrm{k} 3 ; \mathrm{k} 4 ; \mathrm{k} 5$; the number indicates the position of the path in respect of the minimum travel time in congested conditions. Routes have been ordered considering the congested travel time estimated by means of the computer simulation. In Table 1, characteristics of routes composing the joint choice set are summarized. Moreover, the percentage of similarity between pairs of alternatives is reported, both calculated in terms of length and in terms of travel times.

Figure 1, 2 and 3 depict the choice set evolution respectively of group 1, 2 and 3 figure 4 shows the joint choice set evolution.

Table 1: $\quad$ Routes composing the joint choice set.

\begin{tabular}{|c|c|c|c|c|c|c|c|c|c|c|c|c|}
\hline \multirow[t]{2}{*}{ Route } & \multirow[t]{2}{*}{$\begin{array}{l}\text { Length } \\
(\mathrm{Km})\end{array}$} & \multirow[t]{2}{*}{$\begin{array}{l}\text { Congested } \\
\text { travel time }\end{array}$} & \multicolumn{5}{|c|}{$\begin{array}{c}\text { Similarity } \\
\text { (\% length) }\end{array}$} & \multicolumn{5}{|c|}{$\begin{array}{c}\text { Similarity } \\
(\% \text { congested travel time })\end{array}$} \\
\hline & & & k1 & k2 & k3 & k4 & k5 & k1 & k2 & k3 & k4 & $\begin{array}{l}\mathbf{k} \\
\mathbf{5}\end{array}$ \\
\hline k1 & 2.181 & 5'30', & 100 & 40.9 & 53.3 & 40.9 & 80.4 & 100 & 35.7 & 48.2 & 35.7 & 79.6 \\
\hline k2 & 2.116 & $5^{\prime} 50^{\prime \prime}$ & 40.9 & 100 & 44.8 & 87.7 & 44.8 & 35.7 & 100 & 39.8 & 85 & 39.8 \\
\hline k3 & 2.283 & 6'00' & 53.3 & 44.8 & 100 & 44.5 & 72.5 & 48.2 & 39.8 & 100 & 37.7 & 80.8 \\
\hline k4 & 2.293 & 6'00"' & 40.9 & 87.7 & 44.5 & 100 & 41.8 & 35.7 & 85 & 37.7 & 100 & 37.7 \\
\hline k5 & 2.355 & 6'10', & 80.4 & 44.8 & 72.5 & 41.8 & 100 & 79.6 & 39.8 & 80.8 & 37.7 & 100 \\
\hline
\end{tabular}




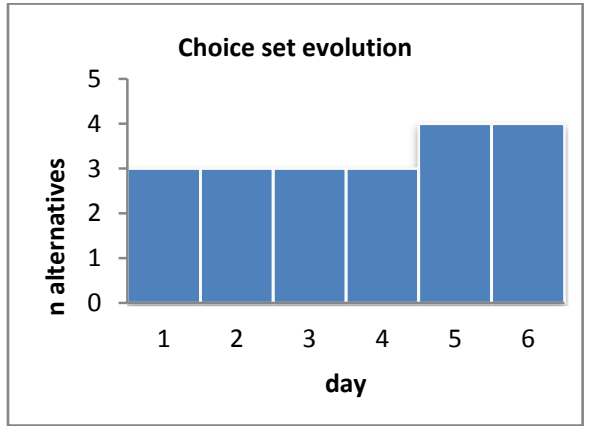

Figure 1: Choice set evolution group 1.

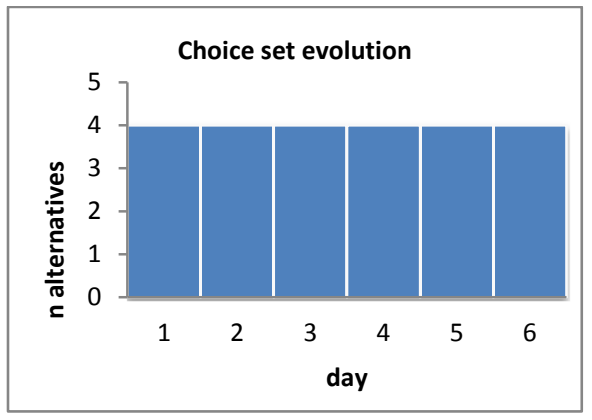

Figure 3: Choice set evolution group 3.

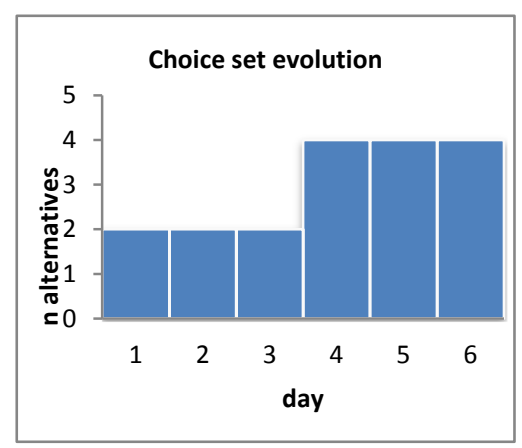

Figure 2: Choice set evolution group 2 .

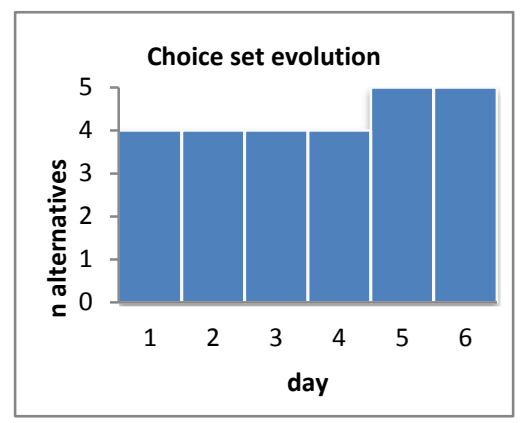

Figure 4: Joint choice set evolution.

Figures 1, 2 and 4 show that choice set is evolving. On the contrary it seems that the choice set of group 3 has not day-to-day changes (Figure 3) and group 3 have a larger choice set since the beginning, if compared with other groups. Considering that the choice set obtained at group level is obtained as a union of choice sets perceived by each single user belonging to the group, the absence of choice set evolution at group level does not mean that there is no evolution of choice set at individual level. According to this conclusion, the absence of additional information decreases the evolutionary trend of the choice set of group 1.

\subsection{Route choice influenced by information}

In order to represent route choice at group level, the percentage of choice per each alternative every day was represented in Figures 5, 6, 7 and 8 respectively for group 1, 2, 3 and joint.

Users of group 1 perceive two routes: $\mathrm{k} 2$ and $\mathrm{k} 3$. The graph in Figure 5 reveals that alternative $\mathrm{k} 2$ is preferred in the last days to alternative $\mathrm{k} 3$. Currently the travel time of alternative $\mathrm{k} 2$ is equal to 5 ' 50 ', whereas the travel time of 
alternative $\mathrm{k} 3$ is equal to 6' 00 ', as shown in Table 1 . Hence, alternative $\mathrm{k} 2$ is characterized by a shorter travel time than alternative $\mathrm{k} 3$. This could mean that users' awareness of the network characteristics is increasing day-to-day.

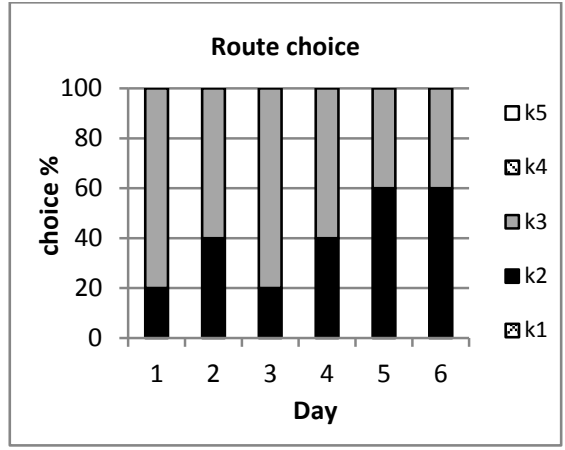

Figure 5: Route choice percentage group 1 .

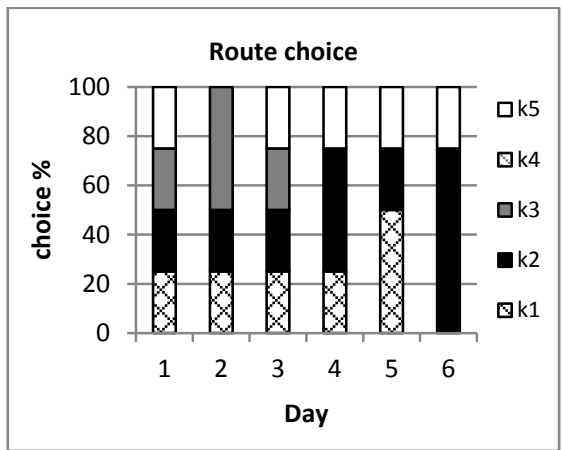

Figure 7: Route choice percentage Figure 8: group 3.

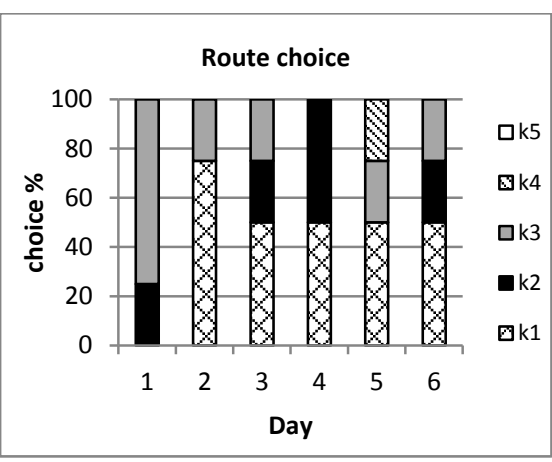

Figure 6: Route choice percentage group 2.

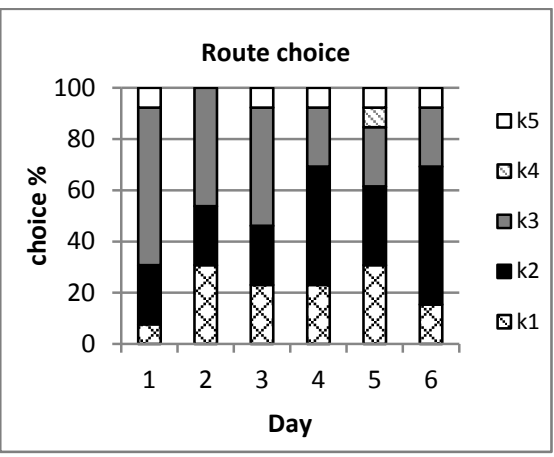

Figure 8: Route choice percentage joint group.

The main difference between figures 5 and 6 is in the number of alternatives chosen by users. In figure 6 appears that day-to-day the number of alternatives chosen increase. This may be due to the additional information users of group 2 receive: because of the information received, users become aware of other alternatives, which enter the choice set and afterwards may be chosen. Moreover, the percentage of users who choose alternative $\mathrm{k} 1$, which is the best, increases day-to-day. This means that a learning process is occurring during the experiment: experience on the network makes users more conscious of the real condition of the network.

In the case of the third group (Figure 7), choices are very variable day-to-day. Alternative k2 is chosen every day. The variability of choice revealed in this graph may be explained considering that wrong information was provided: 
hence, users got confused by information received and are not able to create correct perception of the network. Likely, after a few days, users realized that information obtained were not reliable and, for this reason, decided not to take them into account anymore. This could explain why alternative $\mathrm{k} 5$, which is the worst, is chosen quite every day: users, who are choosing it, not trusting the unreliable information system, prefer to come back to their first choice.

In analysing disaggregate results, users behaviour is correlated to their knowledge of the network: a user who claims a very high knowledge of the network will be not likely to divert, both receiving correct and wrong information. When the user has low knowledge of the network two main situations may occur:

- user carries on choosing the only alternative he knows very well and does not take into account any kind of information;

- user trusts the information obtained and decides to try the new alternative, exploring the network.

The second case occurs often when users have a medium knowledge of the network, sufficient for a reliable exploration.

Figure 8 depicts route choice percentage of the joint group. Alternatives k1 and k2 (the best two paths in term of travel time) choice percentage increases day-byday, whereas route choice percentage of the other alternatives, and in particular of alternative $\mathrm{k} 3$, decrease.

Some comments arise from histograms of figures 5,6 and 7. The learning process, stimulated by the information received, should modify choices, making them converge towards alternatives characterized by minimum travel times, as $\mathrm{k} 1$ and $\mathrm{k} 2$. The convergence process development reveals different characteristics of the three groups, as shown in figures 5-7. The differences between the three convergence processes are analysed in the following.

- In Figure 5, users tend to prefer k2 instead of k1. Perhaps this phenomenon occurs both because route $\mathrm{k} 2$ uses some of the main roads of the network and because users have no additional information. Therefore, if they are satisfied enough of their day before choice, they will not change their mind, they will not explore the network anymore: they will just confirm their previous choice;

- In Figure 6, a quick convergence towards the set composed of $\mathrm{k} 1$ and $\mathrm{k} 2$ is shown: this may be because users feel that information received are reliable, which make easier to users identifying the best alternatives available;

- In Figure 7, the convergence towards the set composed of $\mathrm{k} 1$ and $\mathrm{k} 2$ is also present but slower than its occurrence in Figure 6 . The main reason for the change in convergence speed could be due to users understanding that information is unreliable; in other words, users belonging to group 3 need to explore the network by themselves in order to become aware of the real travel times.

The compliance of users to information received is compared in figures 9 and 10 , considering two users characterized by a medium knowledge of the network (level 2), belonging to group 2 and group 3 respectively. In figure 9 and 10, every day both the experienced travel time on the alternative chosen the day 
before and the information provided on the alternative route are reported. The alternative chosen is the one represented in the fair square, while the alternative presented in the black square is the non-chosen.

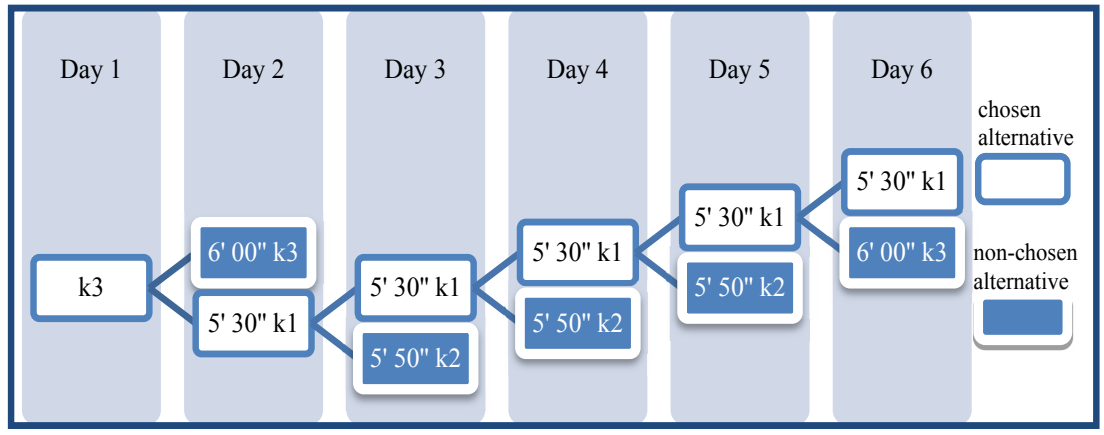

Figure 9: $\quad$ Compliance of group 2.

Figure 9 reveals that user of group 2 changes route as soon as he receives a piece of information on an alternative with lower travel time. Considering that the experienced travel time on day 2 on route $\mathrm{k} 1$, confirms the information received the day before on route $\mathrm{k} 1$, the user continues choosing alternative $\mathrm{k} 1$ during all the following days.

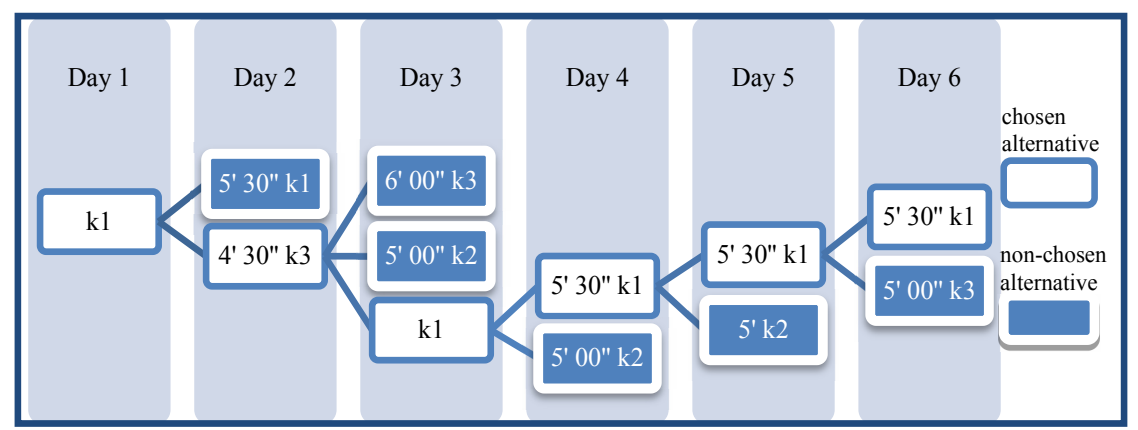

Figure 10: $\quad$ Compliance of group 3.

In Figure 10, it is shown that, at the beginning of the process (day 2) user compliance to information received is very high because he changed his mind preferring route $\mathrm{k} 3$. On day 3 , because the experienced travel time on route $\mathrm{k} 3$ (6' $00^{\prime}$ ') is higher than the forecasted travel time on route $\mathrm{k} 3$ the day before ( 4 ' 30 '), the user does not accept the new information proposing route k2 (forecasted travel time 5, 00 ', ) and prefers to come back to his initial choice. From then on, the user will not use the information received anymore, continuing to choose the alternative $\mathrm{k} 1$, which was his favourite since the beginning.

Some conclusions can be drawn observing the day-to-day processes of other users: for example, it seems that users characterized by a very high level of 
knowledge of the network (level 5) will not change their mind whatever sort of information (right or wrong) they receive. As a consequence, it is possible to affirm that the knowledge of the network plays a key role on users' compliance to information.

\subsection{Switching behaviour}

The switching behaviour is analysed in this section. Figure 11 shows the percentage of switching occurring every day into each group.

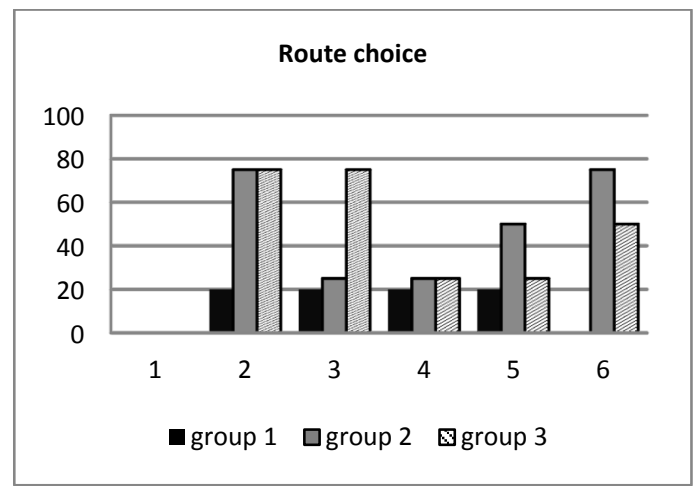

Figure 11: Route switching percentage.

Figure 11 reveals that the highest percentage of switching in registered in the second day. Likely, this is due to information received by users, who decide to change their mind. Moreover, group 1 is not very likely to change route. Indeed, users belonging to group 1 receive only information on the experienced travel time on the route chosen the day before, and for this reason, particularly if they have a not high knowledge of the network, they have not any stimulation to change. On the contrary both group 2 and group 3 are stimulated to change and this effect is probably due to additional information received.

\section{Conclusions}

In this paper a day-to-day experiment carried out on the road network of the town of Reggio Calabria was described and preliminary results were commented through some descriptive statistics. Both choice set and route choice evolution were investigated. Some restrictive assumptions usually introduced in several works available in literature were eliminated: cooperation was considered as a physiological phenomenon involved in the experiment, choice set was dynamic, a new alternative entering the choice set may also be never chosen.

Preliminary results of the descriptive analyses were commented in section 4 and everything found a quite reasonable explanation. Users' characteristics attributes helped to make some results clearer. 
This kind of experiment, taking into account the influence of information provided to users, could provide us with more insights in users' switching behaviour. Afterwards, a behavioural model, able to simulate users' behaviour day by day, could be specified. Knowing users' perception of the network and consequential choices may help the analysts to define some good practices, improving traffic management. These findings may be used also in evacuation conditions, modifying the model in order to make it suitable to an emergency environment. Moreover, in a smart city information may be provided to users easily and in this way route guidance may be diffused quickly and efficiently. Obviously, this could be helpful in evacuation conditions.

In order to obtain some more findings, disaggregate results should be more investigated. In addition to this the experiment should be repeated for a longer time period, in a real network, monitoring users' choices through GPS technology. In the end, a mathematical model should be specified and calibrated using data collected through the real network survey.

\section{Acknowledgement}

Partially supported by national MIUR under PRIN2009 grants n. 2009EP3S42_001.

\section{References}

[1] Quattrone A. and Vitetta A., Random and fuzzy utility models for road route choice. Transportation Research Part E: Logistics and Transportation Review, 47(6), pp. 1126-1139, 2011.

[2] Watling D., Vuren T., (1993), The modelling of dynamic route guidance systems, Transportation Research part $C$ vol. 1, No. 2, pp. 159-182.

[3] Bifulco G. N., Cantarella G. E., de Luca S., Di Pace R., (2011), Analysis and modelling the effects of information accuracy on travellers' behaviour, 14th International IEEE Conference on Intelligent Transportation Systems.

[4] Horowitz, J. L., (1984), The stability of stochastic equilibrium in a two-link transportation network. Transportation Research Part B: Methodological, 18(1): 13-28.

[5] Cantarella G. E., (1993), Day-to-day dynamic in transportation networks: stability and limits of equilibrium in a two-link network, Sistemi Urbani gennaio-aprile.

[6] De Maio M.L., Musolino G., Vitetta A, The role of ITS in evacuation route choice. WIT Transactions on Information and Communication Technologies, 44, pp. 503-515, 2012.

[7] Russo F. and Chilà G., The role of the informer in user behaviour. WIT Transactions on Ecology and the Environment 155, pp. 1027-1044, 2011.

[8] Russo F., Rindone C., Trecozzi M. R., The role of training in evacuation. WIT Transactions on Information and Communication Technologies 44, pp. 491-502, 2012. 
[9] Russo F. and Chilà G., Safety of users in road evacuation: Demand models. WIT Transactions on the Built Environment 96, pp. 773-782, 2007.

[10] Vitetta A., Musolino G. and Marcianò F.A., Safety of users in road evacuation: Calibration of cost functions and simulation. WIT Transactions on the Built Environment 101, pp. 715-725, 2008.

[11] De Maio M.L., Musolino G., Vitetta A, Traffic assignment models in road evacuation. WIT Transactions on Ecology and the Environment, 155, pp. 1041-1051, 2011.

[12] De Maio M. L., Fuzzy Utility Models: possible applications in evacuation conditions in smart cities. WIT Transactions on Ecology and the Environment, 173, pp. 779-790, 2013.

[13] Russo F. and Chilà G., A prototypal test using stated preferences to model evacuation decisions. WIT Transactions on Ecology and the Environment, 173, pp. 743-752, 2013.

[14] Russo F. and Chilà G., Dynamic approaches to demand model in evacuation conditions. WIT Transactions on the Built Environment, 111, pp. 303-312, 2010.

[15] Russo F. and Chilà G., A sequential dynamic choice model to simulate demand in evacuation conditions. WIT Transactions on Information and Communication Technologies, 43 Part I, pp. PI431-PI442, 2010.

[16] Iida Y., Akiyama T., Uchida T., (1992), Experimental analysis of dynamic route choice behaviour. Transportation Research part B vol. 26B No. 1, pp. 17-32

[17] Jha M., Madanat S., Peeta S., (1998), Perception updating and day-to-day travel choice dynamics in traffic networks with information provision. Transportation Research part C vol. 6, pp. 189-212.

[18] Mahmassani H., Liu Y., (1999). Dynamics of commuting decision behavior under advanced traveller information systems. Transportation Research Part C, 7 (2/3), pp. 91-107.

[19] Meneguzzer C., Olivieri A., (2013), Day-to-day traffic Dynamics: laboratory like experiment on route choice and route switching in a simple network with limited feedback information. Forthcoming Procedia-social and behavioural science.

[20] Giffinger R., Christian F., Hans K., Robert K., Nataša P.-M., Evert M., Smart cities - Ranking of European medium-sized cities. http://www.smartcities.eu/. Vienna: Centre of Regional Science, 2007.

[21] Praticò F.G., Casciano, A., Tramontana, D., Pavement life-cycle cost and asphalt binder quality: Theoretical and experimental investigation. Journal of Construction Engineering and Management, Volume 137, Issue 2, pp. 99-107, 2011. 